\title{
Insomnia is Associated with Depression and Anxiety in Patients Undergoing Noncardiac Surgery
}

\section{Xiao Huang}

Beijing Chaoyang Hospital

Changwei Wei

Beijing Chaoyang Hospital

Jing Wang

Beijing Chaoyang Hospital

Dandan Lin

Beijing Chaoyang Hospital

Juxia Zhang

Beijing Chaoyang Hospital

Yanan Hao

Beijing Chaoyang Hospital

Dan Wu

Beijing Chaoyang Hospital

Anshi Wu ( $\nabla$ wuanshi99@163.com )

Beijing Chaoyang Hospital https://orcid.org/0000-0001-7239-3139

\section{Research article}

Keywords: insomnia, depression, anxiety, cognition, postoperative pain, noncardiac surgery

Posted Date: June 9th, 2020

DOI: https://doi.org/10.21203/rs.3.rs-32571/v1

License: (c) (i) This work is licensed under a Creative Commons Attribution 4.0 International License.

Read Full License 


\section{Abstract}

\section{Background}

To investigate the difference of demographic, health status and clinical characteristics of patients with or without insomnia postoperatively, and to identify the potential risk factors of insomnia.

Methods

299 patients undergoing surgery were included. Patients were divided into group $A$ (insomnia, $N=78$ ) and group B (without insomnia, N = 221). Insomnia Severity Index (ISI), Patient Health Questionnaire-9 (PHQ9), Generalized Anxiety Disorder (GAD-7), and Montreal cognitive assessment (MoCA) were applied to all patients preoperatively. Visual Analogue Scale (VAS) was assessed preoperatively, and at the end of the surgery, one day after surgery, two days after surgery and three days after surgery. The PHQ-9, the GAD-7 and the ISI were reassessed three days after surgery. Information on sociodemographic variables and demographic data were collected.

Results

Among the two groups, the average points patients got in the ISI, PHQ-9 and the GAD-7 in group A were also significantly higher than those in group B. The VAS score 3 days after surgery was significantly higher in group A. The PHQ-9 and the GAD-7 3 days after surgery showed significantly higher depression and anxiety scores in group A. Logistic regression showed ISI $(P<0.001,95 \% \mathrm{Cl}=1.218-1.500)$, the GAD-7 $(P=0.01,95 \% \mathrm{Cl}=0.712-0.954)$ preoperatively and the PHQ-9 postoperatively $(P<0.001,95 \% \mathrm{Cl}=1.226-$ 1.555) were risk factors of insomnia.

\section{Conclusions}

Insomnia is common in patients, which worsen after surgery. The present study suggests that depression and anxiety are risk factors of poor sleep quality after surgery. There is a need for further research and strategies for depression and anxiety management to achieve better sleep and significant health benefits in these patients.

Trial registration: clinical trial, NCT04027751. Registered 22 July

2019, https://clinicaltrials.gov/ct2/show/NCT04027751?

cond $=$ NCT04027751\&cntry $=$ CN\&draw $=2 \&$ rank $=1$.

\section{Background}

Sleep problems are important and damaging comorbidities [1]. Sleep disturbances can influence mood and emotional or behavioral states and are complicated in psychopathology. Significant strong partial associations of the sleep disturbance with anxiety, depression and poor sleep quality and a modest partial association with sleepiness were detected [2]. Insomnia is one of the most common sleep 
disorders and a known risk factor for the incidence and severity of depression, recurrence of depressive episodes and even suicide [3]. Insomnia is common in patients during the perioperative period and produces negative consequences on postoperative recovery [4], such as pain, fatigue, then reducing the quality of life [5], especially in those with tumor surgery [6].

Although Insomnia is one of the most prevalent health problems, it's often neglected and clinicians may be reluctant to address insomnia for the reason of its many potential causes, unfamiliarity with behavioral treatments. Little is known about the sleep condition during the perioperative period. Few studies have focused on possible associations between sleep disorders and clinical characteristics in patients undergoing surgery. There is a need to address sleep disturbances routinely in clinical practice and screening for sleep problems is indicated. Further studies are warranted concerning factors that contribute to sleep disturbances and how they best can be treated. Due to the potential impact of insomnia, the main aim of the present study was to investigate the difference of demographic, health status and clinical characteristics of patients with or without insomnia postoperatively, and to identify the potential risk factors of insomnia.

\section{Method}

\section{Participates}

The study was a retrospective cohort study (clinical trial registration number: NCT04027751). We carefully selected participants from the Beijing Chaoyang Hospital, Capital Medical University. Recruitment started in September 2019 and was completed in March 2020. The study was approved by the Regional Ethical Review Committee at the hospitals and followed the Declaration of Helsinki (2019ke-273).

Participants were recruited according to the following inclusion criteria: aged between 18 to 85 years older, able to read, write and understand; willing and able to complete pre and postoperative surveys; were admitted and had surgery in Beijing Chaoyang Hospital. Exclusion criteria included the following: diagnosed with sleep disorders previously and had a history of medication for that; using antidepressant or antipsychotic drugs; using sedatives preoperatively; ambulatory, non-general anesthesia surgery or cancellation of surgery; a history of substance abuse; history of prescription or supplemental sleeping aids; clinical (such as mucositis, severe pain, nausea, dyspnea, vomiting) and emotional (crying, apathy, aggression) conditions, which would prevent participates from taking part in an interview; did not consent to fill out questionnaires. These were evaluated from the interview at the moment of the data collection.

The researcher asked the potential participants if they felt comfortable answering questions at that time, and informed them that they were free to refuse participation. All patients signed a written consent to participate in this research. The participants were interviewed one day before the operation, end of the operation, two days after the operation and three days after the operation. A sample of 299 patients was enrolled. Patients were divided into two groups according to whether they had insomnia postoperatively, group A (insomnia, $\mathrm{N}=78$ ) and group $B$ (without insomnia, $\mathrm{N}=221$ ). This study tried to explore several 
research questions regarding sleep condition preoperative and depression, anxiety, cognition and as well as the surgical prognosis.

\section{Tools}

Additionally, The presence of sleep disturbances was evaluated by the Insomnia Severity Index (ISI). Sleep continuity was included with objective measures. The ISI is a tool for measuring sleep impairment which permits unrestricted use and distribution. The items are scored from 0 to 28 , with higher scores indicating worse sleep quality. The ISI contains seven items on sleep problems (i.e. trouble falling asleep, waking up during the night, waking up earlier than planned, and troubles getting back to sleep) and sleeps quality during the past month (scale $0-4$ ). ISI is a valid screening instrument for detecting insomnia among patients [7], and we defined insomnia as a score of 8-28 (mild: 8-14; moderate-severe: 15-21; severe: 22-28).

Patient Health Questionnaire (PHQ)-9 is a tool to screen and diagnose the degree of depression. It includes 9 items and is used to measure the sense of how people view their life and how they use their resistance resources to maintain and develop their health. Each item of the PHQ-9 is rated on a 4-point scale, for a total score ranging from 0 to 27 . Higher scores indicate increased severity of symptoms and an increased likelihood of major depressive disorders. The PHQ-9 is designed validity and utility to diagnose depression and is efficient to use $[8,9]$.

Generalized Anxiety Disorder (GAD) -7 is a widely used measure of the worry characteristics of anxiety disorders and is being increasingly used in research and clinical practice [10]. Patients rate their frequency of symptoms within the last two weeks on a four-point scale ranging from "not at all" to "almost every day". The GAD-7 consists of 7 items and ranges from 0 to 21 with higher scores indicating higher GAD symptomatology. The GAD-7 scale is an adequate diagnostic accuracy, validated, brief, a selfadministered tool to screen, rate, and monitor the outcome of anxiety disorders in primary healthcare setups [11].

We used Montreal Cognitive Assessment (MoCA) to screen of cognitive disorder. It involves attention, concentration, executive function, memory, language, etc. The MoCA includes 11 items and ranges from 0 to 30 . The MoCA is more sensitive than the MMSE in detecting mild cognitive impairment and it more extensively tests executive function [12].

Visual Analogue Scale (VAS) is used to evaluate the severity of pain in patients, with the lowest point of 0 as "no pain at all" and the highest points of 10 as "my pain is as bad as it could be". The VAS has been proved to be an effective and reliable method to evaluate acute and chronic pain especially in elderly patients.

The PHQ-9, the GAD-7, the ISI and the MoCA were assessed at the baseline. The VAS was assessed preoperatively, at the end of the surgery, one day after surgery, two days after surgery and three days after surgery. The PHQ-9, the GAD-7 and the ISI were reassessed three days after surgery. 


\section{Data collection}

Patients provided information on sociodemographic variables at enrolment. Demographic data were collected including gender, age, years of education, body mass index (BMI), history of diabetes, hypertension, smoking and drinking, type of surgery, American Society of Anesthesiologists (ASA) classification, operation time, intraoperative urine volume, blood loss, the use of the patient-controlled analgesia, the incidence of postoperative nausea and vomiting (PONV) and the length of hospital stay in addition to the scales above.

\section{Statistical analysis}

Data management and statistical analyses were performed in SPSS Statistics software version 25 (IBM Corp., Armonk, NewYork, United States). The data were presented as means \pm standard error (SEM) for continuous variables if normally distributed, as median (minimum, maximum) if not and as percentages (\%) for categorical variables. An Independent-sample t-test was used for continuous variables and the chisquare test was used for categorical variables. Furthermore, we used an ISI scale score cut-off of 7 for expressing sleep impairment in a clinically applicable way and for quantifying its risks associate with health status and disease severity. The observed distributions in the insomnia were summarized in defined groups based on the scores from ISI as follows: none (0-7), mild (8-14), moderate (15-21), severe (22-28). Depression was rate on PHQ-9 of five levels: none (0-4), mild (5-9), moderate (10-14), moderatesevere (15-19) \severe (20-27). Anxiety was graded according to the score of the GAD-7: none (0-4), mild (5-9), moderate (10-13), moderate-severe (14-18), severe (19-21). And cognition impairment was scaled by the MoCA: none (26-30); mild (18-25), moderate ( $\leq 17)$.

A binary logistic regression analysis was conducted to assess which factors were significantly associated with insomnia. Receiver operator characteristic (ROC) curves were created by plotting sensitivity against 1 -specificity and assessing the total area under the curve for each scale. A P value less than 0.05 in two sides was adopted as the critical level for all tests. All participants provided written informed consent and ethical approval was provided by the local medical ethics committee of the Beijing Chaoyang Hospital, Capital Medical University.

\section{Results}

A total of 299 patients (female $=165$, male $=134$ ) with a mean age of 55 years were evaluated in this study. The demographic data of all participates were shown in Table 1. They were not different concerning demographic characteristics (all $P>0.05$ ). The average points patients got in the ISI, PHQ-9 and the GAD-7 in group A were also significantly higher than those in group $\mathrm{B}(P<0.05)$. Baseline classifications of insomnia, depression, anxiety and cognitive impairment according to the scales was shown in Table 2. There were 46 patients diagnosed with mild insomnia, with 29 (37.3\%) in group A and $17(7.7 \%)$ in group $B$, and $10(12.8 \%)$ patients diagnosed with moderate insomnia $(P<0.05)$. Among the two groups, the number of patients diagnosed with depression and anxiety in group $A$ was also significantly increased compared with that in group $B(P<0.05)$. (Table 2$)$ 
From Table 3, there were no significant differences in intraoperative clinical information between patients with and without insomnia. No significant difference was found in the type of surgery, ASA classification, intraoperative urine volume, blood loss, operation time and the VAS scores at the end of the surgery (all $P$ $>0.05)$.

Table 4 shows the postoperative variables. The VAS scores 3 days after surgery was significantly higher in group $\mathrm{A}(P<0.05)$, but no significant difference was found in the VAS scores in any other time points between the two groups. When comparing patients with insomnia to controls, the PHQ-9 and the GAD-7 3 days after surgery showed significantly higher depression and anxiety scores in group $\mathrm{A}(P<0.05)$. The two groups were matched for postoperative analgesia, the incidence of the PONV and the length of hospital stay.

We used logistic regression to adjust for the scale scores including the ISI of the baseline, the VAS scores 3 days after surgery, and the pre and postoperative PHQ-9 and GAD-7. A significant difference was also found in the ISI $(P<0.001,95 \% \mathrm{Cl}=1.218-1.500)$, the GAD-7 $(P=0.01,95 \% \mathrm{Cl}=0.712-0.954)$ preoperatively and the PHQ-9 $(P<0.001,95 \% \mathrm{Cl}=1.226-1.555)$ postoperatively. (Table 5$)$ Analysis of the area under the curve for the ROC of the tests demonstrated PHQ-9 3 days after surgery was best for detecting insomnia (area under the curve $[\mathrm{AUC}]=0.818$ ). (Fig. 1)

\section{Discussion}

This study was undertaken to observe perioperative clinical data of postoperative insomniacs and to examine predictors of poor sleep quality in patients after surgery and the relationships between sleep, depression and anxiety. Sleep quality was quantified using the validated ISI instrument in the present study. The patients in our study exhibited poor sleep quality, with $18.7 \%$ of the patients before surgery and $26.1 \%$ of the patients after surgery had insomnia. We can see even worse sleep postoperatively. We also found anxiety preoperatively and depression postoperatively were risk factors of insomnia.

Research showed insomnia was highly prevalent and associated with unhealthy physical and mental condition [13]. Our study provides support that patients suffering from anxiety and depression were more likely to experience insomnia after surgery. Importantly, our study reveals that this association in patients with the operation. Anxiety and depression have often been showed to be the primary risk factors of depression. Sleep disturbance was highly prevalent in patients, and the high prevalence of sleep disorder and its association with psychological outcomes may have poor implications and may impede cardiac rehabilitation efforts [14]. A meta-analysis indicates that short and long sleep duration was significantly associated with increased risk of depression in adults [15]. Depression and anxiety in elderly Asian participates are associated with several sleep disorders by somewhat different profile [16]. Although the relationship between insomnia and depression has been studied extensively [17], little is known about the association between sleep quality and depression or between insomnia and anxiety in surgical patients.

Similar to our findings, a study showed depression and symptoms related to menopause were independent predictors for poor sleep quality [18]. Overall, patients with sleep disturbances were more 
likely to develop anxiety compared to those without a history of sleep disturbances. Insomnia may be a key pathology in anxiety-related disorders. But we are not able to assign causal directionality to the associations between poor sleep quality and disease characteristics in these patients. Although poor sleep and depression and anxiety seem to be strongly related, it is not elucidated if exacerbations cause poor sleep or if poor sleep contributes to exacerbations. And the biological processes underlying this causal relationship are yet not fully understood. Patients with insomnia present fragmented REM sleep, which probably interferes with basal processes of emotion regulation, and this interaction could lead to depression. Although preliminary results seem to support this hypothesis, data are still too few to make valid conclusions. However, to address this issue, more studies are needed in a longitudinally-followed population sample of patients.

Inconsistent to our research, Ju et al. reported that sleep duration and sleep quality may play a role in cognitive performance in healthy older adults [19]. Findings support a role for sleep disturbances in the development of cognitive impairment $[20,21]$ and suggest sleep plays a crucial part in brain aging. But we didn't find any significant difference in the relationship between cognition and insomnia. And more research is needed to confirm the potential link. We also haven't found strong evidence that insomnia was related to pain. Our study was consistent with a study by Chen et al. [22], which indicates no correlation between sleep quality and pain. Previous research has suggested a relationship between the experience of pain and sleep disruptions among patients undergoing surgery, such that more severe pain is associated with more frequent awakenings throughout the night [23]. The possible reason for the different conclusions may be that we only observed pain and insomnia within 3 days after surgery, and the long term condition was not known.

The findings in the present study are in broad agreement with previous studies, which have reported that poor sleep quality was associated with poorer health status, disease severity, impaired daily activities, and reduced survival $[24,25]$. This study acts as a reminder to enquire about sleep quality in patients with the operation and consider investigation and treatment for potentially treatable cause, including measures to improve factors that adversely affect sleep. And our study underlines the need for specific recommendations for the management of poor sleep quality in patients to achieve significant health benefits and vice versa. Preoperative counseling, early postoperative sleep modifications, and possibly preemptive use of medications may improve transient sleep disturbance among surgical patients. There is a need to manage sleeping disturbance routinely in clinical practice and screen for sleeping problems. Further studies are warranted concerning factors that contribute to sleep disturbance and how they best can be treated. This study makes an important contribution to the literature in that it extends the existing evidence linking insomnia to depression and anxiety. Insomnia has been considered the forgotten part. As a high percentage of patients are affected, they are clinically significant.

The implications for the prevention and treatment of anxiety-related disorders are needed. Doctors must manage patients' expectations while working with them to optimize sleep quality. Several 'biopsychosocial' mechanisms hold promise as possible treatment targets for sleep interventions to reduce both insomnia and symptoms [26]. Cognitive behavior therapy were both effective at reducing 
insomnia and depression severity for older adults. Mental health services were proved to improve recovery outcomes for older adults with depression [27]. It is important to design interventions aimed at decreasing the effects of poor sleep quality in surgical patients to support improving their health and quality of life. The ever increasing prevalence rate of sleep disorders, stresses the utmost importance to identify modifiable risk factors. As poor sleep quality exists in so many patients during the perioperative stage, correctly diagnosing and treating sleep problems in individuals is important. Timely and effectively managing sleep problems is highly relevant from a clinical standpoint, as they can exacerbate specific associated symptoms, such as inattention or irritability.

We note there are several limitations to our study. This study was based on data from a large prospective study, so additional clinical data related to sleep disorders such as sleep duration time were not collected fully. Moreover, we only record clinical information within 3 days after surgery, and the long-term postoperative complications were not observed. Finally, we didn't record the sleep conditions of other patients in the same ward, which may have a great influence on the participants.

Insomnia is common in patients, which worsen after surgery. The present study suggests that depression and anxiety are risk factors of poor sleep quality after surgery. To conclude, our findings suggest a link between sleep impairment in participates and worse health status. The finding is of high clinical relevance. Since patients with operation often report poor sleep quality, there is a need for further research and strategies for depression and anxiety management to achieve better sleep and significant health benefits in these patients.

\section{Abbrevations}

ISI: Insomnia Severity Index; PHQ: Patient Health Questionnaire; GAD: Generalized Anxiety Disorder; MOCA: Montreal Cognitive Assessment; VAS: Visual Analogue Scale; BMl: body mass index; ASA: American Society of Anesthesiologists; PONV: postoperative nausea and vomiting; ROC: Receiver operator characteristic

\section{Declarations}

\section{Acknowledgements}

Not applicable.

\section{Authors' contributions}

Conception and design, critical revision for this study protocol: Anshi Wu, Changwei wei and Xiao Huang. Recruiting subjects: Dan Wu. Test performed and data collection: Jing Wang, Dandan Lin, Yanan Hao and Juxia Zhang. Data analysis: Xiao Huang. The manuscript was wrote by Xiao Huang. All authors read and approved the final manuscript. 


\section{Funding}

This study was funded by Beijing Hospitals Authority Youth Programme(QML20190307) and The Youth Backbone of Beijing Outstanding Talent Training Project (2018).

\section{Availability of data and materials}

Data and materials can be obtained by contacting the corresponding author.

\section{Ethics approval and consent to participate}

The study was approved by the Ethics Committee of Beijing Chaoyang Hospital, Capital Medical University. All procedures performed in studies involving human participants were following the ethical standards of the institutional and/or national research committee and with the 1964 Helsinki declaration and its later amendments or comparable ethical standards. Informed consent was obtained from all individual participants included in the study.

\section{Consent for publication}

Not applicable.

\section{Competing interests}

The authors declare that they have no competing interests.

\section{References}

1. lerodiakonou D, Bouloukaki , Kampouraki M, Papadokostakis P, Poulorinakis I, Lampraki I, et al. Subjective sleep quality is associated with disease status in COPD patients. The cross-sectional Greek UNLOCK study. Sleep Breath. 2020. https://doi.org/10.1007/s11325-020-02039-8.

2. Ozdemir PG, Boysan M, Selvi Y, Yildirim A, Yilmaz E. Psychometric properties of the Turkish version of the Sleep Hygiene Index in clinical and non-clinical samples. Compr Psychiatry. 2015; 59:135-140. https://doi.org/10.1016/j.comppsych.2015.02.001.

3. Chiara Baglioni, Dieter Riemann. Is Chronic Insomnia a Precursor to Major Depression? Epidemiological and Biological Findings. Curr Psychiatry Rep. 2012; 14 (5): 511-518. https://doi.org/10.1007/s11920-012-0308-5.

4. Manning BT, Kearns SM, BohI DD, Edmiston T, Sporer SM, Levine BR. Prospective Assessment of Sleep Quality Before and After Primary Total Joint Replacement. Orthopedics. 2017; 40(4):e636e640. https://doi.org/10.3928/01477447-20170411-01.

5. Halle IH, Westgaard TK, Wahba A, Oksholm T, Rustøen T, Gjeilo KH. Trajectory of sleep disturbances in patients undergoing lung cancer surgery: a prospective study. Interact Cardiovasc Thorac Surg. 2017; 25(2):285-291. https://doi.org/10.1093/icvts/ivx076. 
6. Induru RR, Walsh D (2014) Cancer-related insomnia. Am J Hosp Palliat Care. 2014; 31(7):777-785. https://doi.org/10.1177/1049909113508302.

7. Gagnon $C$, Bélanger $L$, Ivers $H$, Morin $C M$. Validation of the Insomnia Severity Index in primary care. J Am Board Fam Med. 2013; 26(6):701-710. https://doi.org/10.3122/jabfm.2013.06.130064.

8. Spitzer RL, Kroenke K, Williams JB. Validation and utility of a self-report version of PRIME-MD: the PHQ primary care study. Primary Care Evaluation of Mental Disorders. Patient Health Questionnaire, JAMA. 1999; 282(18):1737-1744. https://doi.org/10.1001/jama.282.18.1737.

9. Arrieta J, Aguerrebere M, Raviola G, Flores H, Elliott P, Espinosa A, et al. Validity and Utility of the Patient Health Questionnaire (PHQ)-2 and PHQ-9 for Screening and Diagnosis of Depression in Rural Chiapas, Mexico: A Cross-Sectional Study. J Clin Psychol. 2017; 73(9):1076-1090. https://doi.org/10.1002/jclp.22390.

10. Dear BF, Titov N, Sunderland M, McMillan D, Anderson T, Lorian C, et al. Psychometric comparison of the generalized anxiety disorder scale-7 and the Penn State Worry Questionnaire for measuring response during treatment of generalised anxiety disorder. Cogn Behav Ther. 2011; 40(3):216-227. https://doi.org/10.1080/16506073.2011.582138.

11. Esser P, Hartung TJ, Friedrich $M$, Johansen $C$, Wittchen $H U$, Faller $H$, et al. The Generalized Anxiety Disorder Screener (GAD-7) and the anxiety module of the Hospital and Depression Scale (HADS-A) as screening tools for generalized anxiety disorder among cancer patients. Psychooncology. 2018; 27(6):1509-1516. https://doi.org/10.1002/pon.4681.

12. Olson RA, Iverson GL, Carolan H, Parkinson M, Brooks BL, McKenzie M. Prospective comparison of two cognitive screening tests: diagnostic accuracy and correlation with community integration and quality of life. J Neurooncol. 2011; 105(2):337-344. https://doi.org/10.1007/s11060-011-0595-4.

13. Roth T, Coulouvrat C, Hajak G, Lakoma MD, Sampson NA, Shahly V, et al. Prevalence and Perceived Health Associated With Insomnia Based on DSM-IV-TR; International Statistical Classification of Diseases and Related Health Problems, Tenth Revision; And Research Diagnostic Criteria/International Classification of Sleep Disorders, Second Edition Criteria: Results From the America Insomnia Survey. Biol Psychiatry. 2011; 69 (6): 592-600.

https://doi.org/10.1016/j.biopsych.2010.10.023.

14. Le Grande MR, Jackson AC, Murphy BM, Thomason N. Relationship between sleep disturbance, depression and anxiety in the 12 months following a cardiac event. Psychol Health Med. 2016; 21(1): 52-59. https://doi.org/10.1080/13548506.2015.1040032.

15. Zhai L, Zhang $H$, Zhang D. sleep duration and depression among adults: a meta-analysis of prospective studies. Depress Anxiety. 2015; 32 (9): 664-670. https://doi.org/10.1002/da.22386.

16. Yu J, Rawtaer I, Fam J, Jiang MJ, Feng L, Kua EH, et al. Sleep Correlates of Depression and Anxiety in an Elderly Asian Population. Psychogeriatrics. 2016; 16 (3): 191-195. https://doi.org/10.1111/psyg.12138.

17. Yang Y, Zhu DM, Zhang C, Zhang Y, Wang C, Zhang B, et al. Brain Structural and Functional Alterations Specific to Low Sleep Efficiency in Major Depressive Disorder. Front Neurosci. 2020; 
14:50. https://doi.org/10.3389/fnins.2020.00050.

18. Mansano-Schlosser TC, Ceolim MF, Valerio TD. Poor sleep quality, depression and hope before breast cancer surgery. Appl Nurs Res. 2017; 34: 7-11. https://doi.org/10.1016/j.apnr.2016.11.010.

19. Ju YE, McLeland JS, Toedebusch CD, Xiong C, Fagan AM, Duntley SP, et al. Sleep quality and preclinical Alzheimer disease. JAMA Neurol. 2013; 70(5):587-593.

https://doi.org/10.1001/jamaneurol.2013.2334.

20. Wang C, Holtzman DM. Bidirectional relationship between sleep and Alzheimer's disease: role of amyloid, tau, and other factors. Neuropsychopharmacology. 2020; 45(1):104-120. https://doi.org/10.1038/s41386-019-0478-5.

21. Walker MP. The role of slow wave sleep in memory processing. J Clin Sleep Med. 2009; 5(Suppl.):S20-S26.

22. Chen AF, Orozco FR, Austin LS, Post ZD, Deirmengian CA, Ong AC. Prospective Evaluation of Sleep Disturbances After Total Knee Arthroplasty. J Arthroplasty. 2016; 31(1):330-332. https://doi.org/10.1016/j.arth.2015.07.044.

23. Herrero-Sánchez MD, García-Iñigo Mdel C, Nuño-Beato-Redondo BS, Fernández-de-Las-Peñas C, Alburquerque-Sendín F. Association between ongoing pain intensity, health-related quality of life, disability and quality of sleep in elderly people with total knee arthroplasty. Cien Saude Colet. 2014; 19(6): 1881-1888. https://doi.org/10.1590/1413-81232014196.04632013.

24. Michael A Grandner, Atul Malhotra. Connecting Insomnia, Sleep Apnoea and Depression. Respirology. 2017; 22 (7): 1249-1250. https://doi.org/10.1111/resp.13090.

25. Castro-Marrero J, Zaragozá MC, González-Garcia S, Aliste L, Sáez-Francàs N, Romero O, et al. Poor self-reported sleep quality and health-related quality of life in patients with chronic fatigue syndrome/myalgic encephalomyelitis. J Sleep Res. 2018; 27(6):e12703. https://doi.org/10.1111/jsr.12703.

26. Matthew J Blake, John A Trinder, Nicholas B Allen Mechanisms Underlying the Association Between Insomnia, Anxiety, and Depression in Adolescence: Implications for Behavioral Sleep Interventions. Clin Psychol Rev. 2018; 63: 25-40. https://doi.org/10.1016/j.cpr.2018.05.006.

27. Sadler P, McLaren S, Klein B, Harvey J, Jenkins M. Cognitive Behavior Therapy for Older Adults With Insomnia and Depression: A Randomized Controlled Trial in Community Mental Health Services. Sleep. 2018; 41 (8). https://doi.org/10.1093/sleep/zsy104.

\section{Tables}


Table 1 Preoperative Patient Characteristics by Status of ISI 3 Days After Surgery

\begin{tabular}{|c|c|c|c|c|}
\hline Variables & All $(\mathrm{N}=299)$ & Group A $(N=78)$ & Group B $(\mathrm{N}=221)$ & $P$-Value \\
\hline Sex-female; $n(\%)$ & $165(55.2)$ & $44(56.4)$ & $121(54.8)$ & 0.8 \\
\hline Age, years & $55.2 \pm 0.8$ & $56.9 \pm 1.5$ & $54.6 \pm 1.0$ & 0.224 \\
\hline Years of education, years & $11.3 \pm 0.2$ & $11.0 \pm 0.4$ & $11.3 \pm 0.2$ & 0.556 \\
\hline Body mass index, $\mathrm{kg} / \mathrm{m}^{2}$ & $24.6 \pm 0.2$ & $24.0 \pm 0.4$ & $24.8 \pm 0.2$ & 0.126 \\
\hline VAS before surgery & $0.5 \pm 0.1$ & $0.6 \pm 0.2$ & $0.4 \pm 0.1$ & 0.281 \\
\hline Diabetes & $52(17.4)$ & $13(16.7)$ & $39(17.6)$ & 0.844 \\
\hline Hypertension & $105(35.1)$ & $27(34.6)$ & $78(35.3)$ & 0.914 \\
\hline Current smoking; $n(\%)$ & $47(15.7)$ & $7(9.0)$ & $40(18.1)$ & 0.137 \\
\hline Alcohol use; $n(\%)$ & $19(6.4)$ & $4(5.1)$ & $15(6.8)$ & 0.712 \\
\hline ISI of baseline & $3.9 \pm 0.2$ & $7.5 \pm 0.6$ & $2.7 \pm 0.2$ & $\square 0.001$ \\
\hline PHQ-9 of baseline & $2.6 \pm 0.3$ & $4.8 \pm 1.1$ & $1.8 \pm 0.2$ & 0.008 \\
\hline GAD-7 of baseline & $2.1 \pm 0.2$ & $3.0 \pm 0.4$ & $1.7 \pm 0.2$ & 0.005 \\
\hline MoCA & $25.3 \pm 0.2$ & $24.8 \pm 0.4$ & $25.5 \pm 0.2$ & 0.117 \\
\hline
\end{tabular}

The data were presented as means \pm standard error, SEM for continuous variables, and t value are presented; as percentages

(\%) for categorical variables and $\chi$ value are presented, VAS Visual Analog Scale, ISI Insomnia Severity Index, PHQ-9 Patient

Health Questionnaire-9, GAD-7 Generalized Anxiety Disorder, MoCA Montreal cognitive assessment

Table 2 Baseline Classification of Scales

\begin{tabular}{|c|c|c|c|c|}
\hline Variables & Patients $(\mathrm{N}=299)$ & Group A & Group B & $P$-Value \\
\hline ISI; $\mathrm{n}(\%) \quad(\mathrm{N}=299)$ & & & & $\square 0.001$ \\
\hline Non & $243(81.3)$ & $39(50)$ & $204(92.3)$ & \\
\hline Mild & $46(15.4)$ & $29(37.2)$ & $17(7.7)$ & \\
\hline Moderate & $10(3.3)$ & $10(12.8)$ & $0(0)$ & \\
\hline PHQ-9; $n(\%)(\mathrm{N}=298)$ & & & & $\square 0.001$ \\
\hline Non & $244(81.9)$ & $49(62.8)$ & $195(88.6)$ & \\
\hline Mild & $38(12.8)$ & $21(26.9)$ & $17(7.7)$ & \\
\hline Moderate & $16(5.4)$ & $8(10.3)$ & $8(3.6)$ & \\
\hline GAD-7; $n(\%) \quad(\mathrm{N}=299)$ & & & & 0.022 \\
\hline Non & $244(81.6)$ & $56(72.8)$ & $188(85.1)$ & \\
\hline Mild & $44(14.7)$ & $17(21.8)$ & $27(12.2)$ & \\
\hline Moderate & $9(3.0)$ & $5(6.4)$ & $4(1.8)$ & \\
\hline Moderate-severe & $2(0.7)$ & $0(0)$ & $2(0.9)$ & \\
\hline MoCA; $n(\%)(\mathrm{N}=296)$ & & & & 0.336 \\
\hline Non & $162(54.7)$ & 37 (48.7) & $125(56.8)$ & \\
\hline Mild & $124(41.9)$ & $35(46.1)$ & $89(40.5)$ & \\
\hline Moderate & $10(3.4)$ & $4(5.3)$ & $6(2.7)$ & \\
\hline
\end{tabular}

Percentages (\%) for categorical variables and $\chi$ value are presented, ISI Insomnia Severity Index, $P H Q-9$ Patient Health Questionnaire-9, GAD-7 Generalized Anxiety Disorder-7, MoCA Montreal cognitive assessment 
Table 3 Intraoperative Variables by Status of ISI 3 Days After Surgery

\begin{tabular}{ccccc}
\hline Variables & All $(\mathrm{N}=299)$ & Group A (N=78) & Group B (N=221) & $P$-Value \\
\hline Type of surgery; $n(\%)$ & & & & 0.117 \\
\hline Thoracic surgery & $67(22.4)$ & $25(32.1)$ & $42(19.0)$ & \\
\hline Orthopaedic surgery & $45(15.1)$ & $14(17.9)$ & $31(14.0)$ & \\
\hline urology & $71(23.7)$ & $15(19.2)$ & $56(25.3)$ & \\
\hline Gynecologic surgery & $45(15.1)$ & $5(6.4)$ & $40(18.1)$ & \\
\hline Gastrointestinal surgery & $28(9.4)$ & $8(10.3)$ & $20(9.0)$ & \\
\hline Others & $43(14.4)$ & $11(14.1)$ & $32(14.5)$ & \\
\hline ASA classification; $n(\%)$ & & & & \\
\hline Class $\square$ & $36(12.0)$ & $7(9.0)$ & $29(13.1)$ & \\
\hline Class II & $190(63.5)$ & $50(64.1)$ & $140(63.3)$ & \\
\hline Class III & $73(24.4)$ & $21(26.9)$ & $52(23.5)$ & \\
\hline Intraoperative urine volume, $\mathrm{mL}$ & $426.8 \pm 22.2$ & $465.0 \pm 50.9$ & $412.4 \pm 23.8$ & 0.291 \\
\hline Intraoperative blood loss, $\mathrm{mL}$ & $110.9 \pm 8.1$ & $104.4 \pm 14.3$ & $113.4 \pm 9.8$ & 0.622 \\
\hline Operation time, min & $160.3 \pm 4.6$ & $159.2 \pm 9.3$ & $160.7 \pm 5.3$ & 0.892 \\
\hline VAS at the end of surgery & $2.4 \pm 0.1$ & $2.6 \pm 0.2$ & $2.3 \pm 0.1$ & 0.371 \\
\hline
\end{tabular}

ASA American Society of Anesthesiologists, VAS Visual Analog Scale

Table 4 Postoperative Variables by Status of ISI 3 Days After Surgery

\begin{tabular}{|c|c|c|c|c|}
\hline Variables & All $(\mathrm{N}=299)$ & Group A $(\mathrm{N}=78)$ & Group B $(\mathrm{N}=221)$ & $P$-Value \\
\hline Postoperative analgesia; $n(\%)$ & & & & 0.1 \\
\hline Non & $93(31.1)$ & $22(39.1)$ & $71(42.6)$ & \\
\hline PCIA & $179(59.9)$ & $44(55.1)$ & $135(49.4)$ & \\
\hline PCIA & $27(9.0)$ & $12(5.8)$ & $15(8.0)$ & \\
\hline VAS 1 day after surgery & $2.7 \pm 0.1$ & $3.0 \pm 0.3$ & $2.6 \pm 0.1$ & 0.144 \\
\hline VAS 2 days after surgery & $2.2 \pm 0.1$ & $2.3 \pm 0.3$ & $2.1 \pm 0.1$ & 0.674 \\
\hline VAS 3 days after surgery & $1.7 \pm 0.1$ & $2.1 \pm 0.2$ & $1.5 \pm 0.1$ & 0.024 \\
\hline PONV 1day after surgery; $n(\%)(\mathrm{N}=294)$ & & & & 0.722 \\
\hline 0 & $258(87.8)$ & $69(88.4)$ & $189(87.4)$ & \\
\hline 1 & $9(3.1)$ & $3(4.3)$ & $6(4.1)$ & \\
\hline 2 & $3(1.0)$ & $0(0)$ & $3(0.9)$ & \\
\hline 3 & $24(8.2)$ & $6(7.2)$ & $18(7.6)$ & \\
\hline PONV 2 days after surgery; $n(\%)(\mathrm{N}=294)$ & & & & 0.506 \\
\hline 0 & $282(95.9)$ & $76(90.6)$ & $206(97.0)$ & \\
\hline 1 & $6(2.0)$ & $2(6.3)$ & $4(1.5)$ & \\
\hline 2 & $1(0.3)$ & $0(0)$ & $1(0)$ & \\
\hline 3 & $5(1.7)$ & $0(0)$ & $5(1.5)$ & \\
\hline ISI 3 days after surgery & $5.0 \pm 0.3$ & $11.6 \pm 0.4$ & $2.7 \pm 0.2$ & $\square 0.001$ \\
\hline PHQ-9 3 days after surgery & $2.7 \pm 0.2$ & $5.7 \pm 0.5$ & $1.7 \pm 0.2$ & $\square 0.001$ \\
\hline GAD-7 3 days after surgery & $1.7 \pm 0.2$ & $3.2 \pm 0.6$ & $1.2 \pm 0.2$ & 0.001 \\
\hline Length of hospital stay, days & $11.4 \pm 0.4$ & $11.3 \pm 0.8$ & $11.5 \pm 0.5$ & 0.903 \\
\hline
\end{tabular}

PCIA patient-controlled intravenous analgesia, PCEA patient-controlled epidural analgesia, VAS Visual Analog Scale, PONV postoperative nausea and vomiting, PONV 0 indicate no nausea or vomiting; 1 indicate nausea; 2 indicate vomiting; 3 indicate both nausea and vomiting, ISI Insomnia Severity Index, $P H Q-9$ Patient Health Questionnaire-9, GAD-7 Generalized Anxiety 
Table 5 Summary of Logistic Regression with ISI 3 Days After Surgery as Dependent Variable

\begin{tabular}{cccccc}
\hline Variables & $\boldsymbol{\beta}$ & Wald & $P$ & OR & 95\%CI \\
\hline ISI of baseline & 0.301 & 32.355 & $\square 0.001$ & 1.352 & $1.218-1.500$ \\
\hline PHQ-9 of baseline & 0.023 & 0.474 & 0.491 & 1.023 & $0.959-1.091$ \\
\hline GAD-7 of baseline & -0.193 & 7 & 0.01 & 0.824 & $0.712-0.954$ \\
\hline VAS 3 days after surgery & 0.033 & 0.133 & 0.715 & 1.034 & $0.866-1.234$ \\
\hline PHQ-9 3 days after surgery & 0.323 & 28.298 & $\square 0.001$ & 1.381 & $1.226-1.555$ \\
\hline GAD-7 3 days after surgery & -0.048 & 0.736 & 0.391 & 0.953 & $0.854-1.064$ \\
\hline
\end{tabular}

ISI Insomnia Severity Index, PHQ-9 Patient Health Questionnaire-9, GAD-7 Generalized Anxiety Disorder-7, VAS Visual Analog Scale

Figures

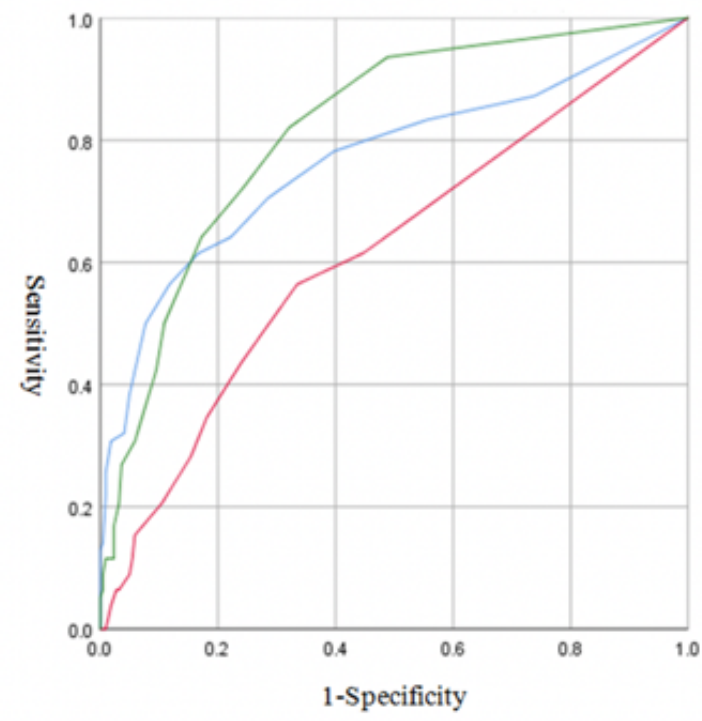

Source of the Curves

- ISI of baseline

GAD-7 of baseline

PHQ-9 3 days after surgery

$R O C$ receiver operator characteristic; ISI Insomnia Severity Index, GAD-7 Generalized Anxiety

\section{Disorder-7, PHQ-9 Patient Health Questionnaire-9}

\section{Figure 1}

ROC Curve of the Risk Factors 